\title{
REDES DE COLABORAÇÃO CIENTÍFICA E ANÁLISE DE POLÍTICAS PÚBLICAS DESPORTIVAS LOCAIS NO BRASIL E EM PORTUGAL
}

\author{
Edmilson Santos dos Santos \\ Universidade Federal do Vale do São Francisco \\ Petrolina - PE. Brasil \\ edmilsonss.univasf@gmail.com \\ Maria José Carvalho \\ Universidade do Porto \\ Portugal \\ mjc@fade.up.pt
}

RESUMO

Objetivo do estudo: O presente trabalho buscou identificar os pesquisadores e os padrões de colaboração na produção científica associada à participação do poder local na oferta de políticas públicas de desporto no Brasil e em Portugal.

Metodologia/abordagem: O procedimento metodológico envolveu revisão sistemática e análise bibliométrica da produção dos pesquisadores. Por último, analisamos as relações entre os atores e as redes de colaboração. Para localização dos trabalhos, utilizamos o Portal Periódicos Capes, que disponibiliza diferentes bases de dados com textos publicados pelos principais editores do mundo, distribuídos em todas as áreas do conhecimento. As redes de colaboração foram observadas via coautorias.

Originalidade/relevância: O poder local no Brasil e em Portugal tem promovido importantes políticas públicas desportivas. No entanto, a análise desta produção, principalmente de forma comparativa entre os dois países, ainda não foi realizada.

Principais resultados: É perceptível o esforço na consolidação da agenda através do aumento do trabalho ao longo do tempo, dos grupos de pesquisadas envolvidos e do número de parcerias interinstitucionais e internacionais. No entanto, a existência de betweenness centrality dificulta a consolidação dessa agenda de pesquisa.

Contribuições teóricas/metodológicas: Promove a discussão da relação entre a produção científica na área de políticas públicas municipais no Brasil e em Portugal e a construção de redes de colaboração.

Palavras-chave: Políticas Públicas. Desporto. Agenda de Pesquisa. Produção Acadêmica.

Cite como

American Psychological Association (APA)

Santos, E. S., \& Carvalho, M. J. (2020, set./dez.). Redes de colaboração científica e análise de políticas públicas desportivas locais no Brasil e em Portugal. PODIUM Sport, Leisure and Tourism Review, São Paulo, 9(Edição Especial), 28-45. https://doi.org/10.5585/podium.v9i4.16461. 


\title{
SCIENTIFIC COOPERATION NETWORKS AND ANALYSIS OF LOCAL PUBLIC SPORT POLICIES IN BRAZIL AND PORTUGAL
}

\begin{abstract}
Objective: The present work sought to identify researchers and patterns of collaboration in scientific production associated with the participation of local authorities in the provision of public sports policies in Brazil and Portugal.

Methodology/ Approach: The methodological procedure involved systematic review and bibliometric analysis of the researchers' production. Finally, we analyze the relationships between the actors and the collaboration networks. To locate the works, we use the Portal Periódicos Capes, which provides different databases with texts published by the main editors in the world, distributed in all areas of knowledge. Collaboration networks were observed via co-authorships.
\end{abstract}

Originality/ Relevance: Local authorities in Brazil and Portugal have promoted important public sports policies. However, the analysis of this production, mainly comparatively between the two countries, has not yet been carried out.

Main Results It is noticeable the effort in the consolidation of the agenda through the increase of work along the time, the groups of researchers involved and the number of inter-institutional and international partnerships. However, the existence of betweenness centrality makes the consolidation of this research agenda difficult..

Theoretical/ Methodological contributions: It promotes the discussion of the relationship between scientific production in the area of municipal public policies in Brazil and Portugal and the construction of collaboration networks.

Keywords: Public Policies. Sport. Research agenda. Academic production.

\section{REDES DE COOPERACIÓN CIENTÍFICA Y ANÁLISIS DE POLÍTICAS LOCALES DE DEPORTE PÚBLICO EN BRASIL Y PORTUGAL}

\section{RESUMEN}

Objetivo: El presente trabajo buscó identificar investigadores y patrones de colaboración en la producción científica asociados con la participación de las autoridades locales en la provisión de políticas deportivas públicas en Brasil y Portugal.

Metodología / Enfoque: El procedimiento metodológico incluyó una revisión sistemática y un análisis bibliométrico de la producción de los investigadores. Finalmente, analizamos las relaciones entre los actores y las redes de colaboración. Para ubicar las obras, utilizamos el Portal Periódicos Capes, que proporciona diferentes bases de datos con textos publicados por los principales editores del mundo, distribuidos en todas las áreas del conocimiento. Las redes de colaboración se observaron a través de coautorías.

Originalidad / Relevancia: Las autoridades locales en Brasil y Portugal han promovido importantes políticas deportivas públicas. Sin embargo, el análisis de esta producción, principalmente comparativamente entre los dos países, aún no se ha llevado a cabo.

Resultados principales: Es notable el esfuerzo en la consolidación de la agenda a través del aumento del trabajo a lo largo del tiempo, los grupos de investigadores involucrados y el número de asociaciones 
interinstitucionales e internacionales. Sin embargo, la existencia de la betweenness centrality dificulta la consolidación de esta agenda de investigación.

Contribuciones teóricas / metodológicas: promueve la discusión de la relación entre la producción científica en el área de políticas públicas municipales en Brasil y Portugal y la construcción de redes de colaboración.

Palabras clave: Políticas públicas. Deporte. Agenda de investigación. Producción académica.

\section{Introdução}

Em diversos países do mundo é possível observar a ampliação das análises que identificam a escala de poder local como importante à oferta de políticas públicas desportivas (Santos, Milani, Reverdito \& Starepravo, 2019; Paipe et al, 2017; Januário, 2010; Ramchandani, Shibli \& Kung, 2018; Hoekman, Roest \& Poel, 2018; Reid, 2018). A maior proximidade do cidadão permite aos decisores locais observarem as preferências dos cidadãos e responderem também às necessidades dos stakeholders locais (clubes, associações, profissionais do desporto, entre outros) de forma a garantir maior responsividade a essas demandas.

O campo de análise de políticas públicas de desporto no Brasil tem produzido alguns trabalhos panorâmicos que permitem avaliar o estágio da produção do conhecimento sobre políticas públicas desportivas locais no Brasil que podem nos ajudar a pensar também na realidade de Portugal. Os trabalhos apresentam uma produção diversificada em termos de foco e de conteúdo e essas análises têm despertado cada vez mais o interesse da comunidade de especialistas $^{1}$ (Bastos, 2003; Santos, Batista \& Araújo, 2007; Starepravo, Afonso \& Ferreira, 2008; Amaral \& Pereira, 2009; Castro et al, 2012; Amaral, Ribeiro \& Silva, 2014; Sousa et al, 2016; Barra, Silva \& Vitorino, 2017). No entanto, tem escapado a elas um olhar específico sobre a produção das análises da participação dos municípios na implementação de políticas desportivas. Em Portugal, ainda não há uma avaliação panorâmica da produção sobre as políticas desportivas.

A inexistência de uma avaliação mais precisa da produção sobre a participação do poder local (subcampo da análise de políticas públicas do desporto) na oferta de políticas públicas desportiva nos dois países suscitou este trabalho, no sentido de analisar os atores responsáveis

\footnotetext{
${ }^{1}$ Consideramos como 'comunidade de especialistas' aqueles pesquisadores que, individualmente ou coletivamente, tenham contribuído com a análise de políticas públicas desportivas, independentemente do volume de produção.
} 
pelas produções e como esses se organizam em comunidades científicas (comunidade de especialistas). Mesmo considerando as diferenças entre Brasil e Portugal no que concerne à forma de governo, com impactos distintos sobre a participação do poder local na oferta deste serviço, é inegável que este tem contribuído para garantir a extensão do direito ao desporto à população nos dois países. Portanto, constitui-se subcampo de investigação profícuo para se compreender as políticas públicas de desporto nos dois países: quem são os pesquisadores e como se organizam em comunidades científicas.

Compreender quem são os atores e como se organizam os pesquisadores da área de políticas públicas desportivas locais no Brasil e em Portugal pode nos ajudar a identificar seu nível de cooperação e colaborar com a construção de uma estratégia que busque maior integração acadêmica entre os dois países.

Assim sendo, o presente estudo buscou identificar os pesquisadores e os padrões de colaboração na produção de conhecimento associado à participação do poder local na oferta de políticas públicas de desporto no Brasil e em Portugal. Como objetivos específicos, buscamos identificar os níveis de cooperação (interpessoal, interinstitucional ou internacional) e de interação entre os pesquisadores (substantiva ou tênue).

\section{Referencial teórico}

Ao imputar como dever do Estado a oferta de políticas públicas desportivas, as duas constituições (do Brasil e de Portugal) garantiram espaço à participação do poder local na oferta da política. Nos dois países, a estrutura de gestão administrativa do Estado incorpora o poder local (prefeituras no Brasil e câmaras municipais em Portugal) como agente implementador de políticas públicas.

Mesmo que os condicionantes normativos da fundação do Estado sejam distintos, os estudos têm demonstrado que nos dois países há uma grande oferta de políticas públicas desportivas por parte do poder local. Enquanto em Portugal há um imperativo normativo exigindo a participação dos municípios (Lei das Autarquias e Lei de Bases da Atividade Física e do Desporto), no Brasil a engenharia federalista não imputou obrigações aos poderes executivos municipais (Santos \& Freitas, 2015).

Passados mais de 30 anos da promulgação das constituições dos dois países, com direito à participação do poder local na extensão do direito ao desporto registrado na norma, é importante avaliar o grau de conhecimento que se tem sobre essa realidade. Ele é dependente 
de como se tem organizado, do ponto de vista da produção científica, os atores e as comunidades que estudam a formulação, a implementação ou a avaliação das políticas públicas desportivas ofertadas pelo poder local.

Portanto, do ponto de vista empírico, há um campo de investigação específico no âmbito das políticas públicas. É possível identificar, na esfera local, o Estado em ação que constitui uma agenda de pesquisa. Nesse caso, é possível identificar não somente um campo disciplinar, nos termos colocados por Kuhn (1976), mas uma agenda específica (Reis, 2003) e a existência de uma comunidade de especialistas (Arretche, 2003).

A ciência tem se desenvolvido em diversas áreas e diferentes países do mundo para ser um empreendimento coletivo (Watanabe, Gomes \& Hoffmann, 2013). O empreendimento coletivo tem permitido agregar pesquisadores que buscam refinamentos teóricos e metodológicos em redes ${ }^{2}$. Portanto, é procedimento cada vez mais comum que pesquisadores se reúnam em redes de relacionamentos para produzirem conhecimento. Essas redes de relacionamento permitem o desenvolvimento de uma área de atuação através de trabalho em parceria com pesquisadores e instituições, o compartilhamento de informações e sua intensidade pode ser aferida, por exemplo, pelo número de artigos publicados (Bulgacov \& Verdu, 2001).

No entendimento de Gatti (2005, p. 124), as redes permitem

A intercomunicação com pares, o trabalho em equipe, as redes de trocas de ideias e disseminação de propostas e achados de investigação, os grupos de referência temática, constituem hoje uma condição essencial à realização de investigações científicas e ao avanço dos conhecimentos.

Bulgacov \& Verdu (2001, p. 166) acrescentam que

A cooperação é um pré-requisito para a inovação, solução de problemas e desempenho. Além disso, as parcerias podem representar corte nas distâncias para o ingresso nas esferas internacionais de investigação, com projetos grandes e pequenos, que estabeleçam parcerias no exterior e no país.

Seja a intercomunicação, seja a cooperação, elas permitem formar redes de colaboração científica que possibilitam maior desenvolvimento da ciência (Balancieri et al, 2005) e refletem a dinâmica do intercâmbio de conhecimento nas áreas (Gazda \& Quandt, 2010). As redes de

\footnotetext{
${ }^{2}$ No entendimento de Silva (2002, p. 2), “As redes científicas são consideradas abertas e ágeis: unem atores múltiplos, toleram mudanças, admitem rearranjos conforme as necessidades".
} 
colaboração científica oportunizam que grupos de pessoas unidas pelos mais variados interesses e motivações possam promover o desenvolvimento de um campo de estudo ${ }^{3}$. Bulgacov $\&$ Verdu (2001) avaliam que essa colaboração abre espaço para novas oportunidades de conhecimento e aprimoramento científico e tecnológico.

A cooperação entre pesquisadores pode dar-se em três níveis institucionais, como destacam Balancieri et al (2005): intrainstitucional, interinstitucional e internacional. A cooperação intrainstitucional é marcada por relações de proximidade, dentro da mesma instituições geralmente dentro de um mesmo setor, departamento, grupo de pesquisa ou na relação orientador-orientando. A cooperação interinstitucional eleva o grau de intercâmbio para além das fronteiras de uma instituição. Ocorre quando pesquisadores ou grupo de pesquisadores de instituições diferentes estabelecem uma agenda comum, podendo ser provisória ou permanente. A cooperação internacional ocorre quando pesquisadores de países diferentes passam a estabelecer relações dentro de um determinado campo da produção ou da divulgação do conhecimento. Ela pode ser considerada como uma variante da cooperação interinstitucional, mas traz como diferencial intercâmbios internacionais.

As relações ainda podem ser substantivas ou tênues. As relações substantivas permitem uma série de conexões. No entanto, todas elas buscam constituir-se como transações valiosas, de proximidade e permanência, compartilhando abordagens teóricas ou procedimentos metodológicos (Bulgacov \& Verdu, 2001). A cooperação acadêmica ocorre na direção do fortalecimento da agenda de pesquisa. Portanto, todos os pesquisadores envolvidos são protagonistas do fazer ciência. A rede é produzida pelo envolvimento voluntário de cada integrante dentro de um dado campo investigativo e são promovidas diferentes formas de colaboração (conforme nota de rodapé 2). Pode ocorrer o compartilhamento de ideias, de instrumentos de pesquisa ou de banco de dados, fortalecendo o elo entre os pesquisadores e as instituições.

A cooperação tênue caracteriza-se por ser esporádica e/ou unidirecional. A cooperação esporádica indica que a produção científica sobre um determinado tema não é prioritária para (pelo menos) um dos integrantes da rede. A cooperação unidirecional indica que há um polo (betweenness centrality) a promover um determinado campo científico. As parcerias gravitam em torno de um personagem. A existência de betweenness centrality tornaria a rede mais

\footnotetext{
${ }^{3}$ Essa colaboração pode dar-se na formação de grupos de pesquisa, participação de bancas ou de cursos em outras instituições, em eventos científicos ou consultorias, conforme Gazda e Quandt (2010).
} 
instável, pois ela seria sensível à retirada desses indivíduos (Soares, Souza \& Moura, 2010). Os colaboradores participam, geralmente, no sentido de garantir o refinamento crítico desse investigador central.

No entanto, a colaboração é dependente da autoria da agenda. A construção de uma agenda de pesquisa inicia com preocupações que são de ordem privada. São muitas agendas disponíveis de investigação e, geralmente, há maior propensão do autor em optar por um número restrito de agenda. Após definição, o autor pode construir diferentes estratégias colaborativas para viabilizar seus estudos.

Quando dois ou mais autores partilham de uma mesma agenda e constituíram o objeto de estudo de forma coletiva, não há ascendência de nenhum sobre a agenda. Esse aspecto é facilmente perceptível quando a autoria é distribuída por ordem alfabética (Gregori Júnior, Godoy \& Gregori, 2012).

$\mathrm{Na}$ maior parte das vezes, o primeiro pode ter tido uma participação fundamental como: (a) autor teve a ideia do estudo, (b) elaborou a metodologia, (c) coordenou a coleta de dados, (d) elaborou a primeira redação do texto, (e) orientou a investigação ou (f) coordenou os trabalhos do grupo de pesquisa (Gregori Júnior, Godoy \& Gregori, 2012; Petroianu, 2003). Portanto, o primeiro autor é um personagem central quando desejamos compreender o desenvolvimento de uma certa agenda de pesquisa.

Uma das formas em que podemos observar de maneira precisa essa colaboração é na difusão do conhecimento nos periódicos científicos. Por diferentes motivos e a partir de diferentes critérios de cooperação, os autores podem envolver-se na produção de um artigo científico. Watanabe, Gomes \& Hoffmann (2013) consideram que coautoria é o principal indicador para se identificar a rede de colaboração. Maia \& Caregnato (2008) identificaram que não só as produções compartilhadas têm aumentado, como também o interesse pela análise dessas colaborações. No âmbito da educação física brasileira, a análise bibliométrica ${ }^{4}$ da produção em políticas públicas de lazer realizada por Onofre, Colângelo \& Lino (2019) chama atenção para esse debate.

No entanto, é preciso considerar que os autores podem ter diferentes tipos de adesão a essa agenda. Desde aqueles que têm relação tênue com esse campo de investigação, que desenvolveram até dois trabalhos no intervalo de 10 anos, que denominamos aqui de

\footnotetext{
${ }^{4}$ A bibliometria é uma área de investigação que se utiliza de técnicas quantitativas e estatísticas para aferir a produção e disseminação do conhecimento científico (Araújo, 2006).
} 
pesquisadores eventuais, e aqueles que desenvolveram acima de três trabalhos no período, classificados aqui de pesquisadores efetivos. Considerando o aspecto colaborativo como importante para o desenvolvimento da ciência, torna-se desnecessário estabelecer esse critério a partir da posição dos autores na produção do artigo.

Portanto, a relação entre pesquisador (autor), colaboradores e obra (artigo) podem produzir diferentes cenários dentro de um determinado campo. Sendo assim, sua análise permite melhor compreensão de como uma comunidade se constitui em termos de dinâmica acadêmica à produção e difusão de artigos científicos.

\section{Metodologia}

Para realizar a investigação dos atores e das redes criadas no Brasil e em Portugal para analisarem as políticas públicas de desporto no âmbito local, organizamos o trabalho em três etapas. Na primeira, realizamos uma revisão sistemática sobre o tema da política pública desportiva local. Na segunda, fizemos a análise bibliométrica da produção dos autores. Por último, analisamos as relações entre os atores e as redes de colaboração.

Para a revisão sistemática, seguimos o mesmo percurso traçado por Sampaio \& Mancini (2007): escolha da base de dados, critérios de inclusão e exclusão dos artigos, verificação da acurácia dos resultados, determinação da qualidade dos estudos. Para localização dos trabalhos, utilizamos o Portal Periódicos Capes, que disponibiliza diferentes bases de dados com textos publicados pelos principais editores do mundo, distribuídos em todas as áreas do conhecimento (CAPES, 2004) e Kocian \& Silva (2016) utilizaram o Portal Periódicos Capes para encontrar trabalhos no âmbito das políticas públicas desportivas de maneira mais ampla, sem filtro quanto ao nível de governo.

Utilizamos a ferramenta Google Acadêmico de forma a corrigir possíveis lacunas dos bancos de dados bibliográficos. A rapidez e a amplitude das informações são pontos positivos ressaltados por Giustini \& Barsky (2005) para a utilização dessa ferramenta de busca que, no entendimento de Shultz (2007), é uma valiosa ferramenta complementar. Esse procedimento se reforça, ainda, tendo em vista que os bancos das áreas de ciências sociais e humanas não são tão extensos no Brasil (Caregnato, 2011).

Fizemos quatro combinações de termos com o operador lógico AND: (1) "esporte"/ “desporto", "município", "políticas públicas"; (2) "esporte”/ "desporto”, "governo local”, “políticas públicas”. (3) “esporte”/ “desporto", “poder local”, “políticas públicas”; (4) 
“esporte"/ "desporto", "município". A utilização alternada da palavra "esporte" e "desporto" foi para contemplar a cultura escrita dos dois países. As seguintes palavras-chave em inglês foram utilizadas, na mesma composição: "sport", "local government", "local power" e "policy".

Como indicadores para triagem dos trabalhos, utilizamos a seguinte estratégia: (a) busca dos termos no ambiente resumo e/ou assunto; (b) com palavras exatas; (c) artigos de revistas; (d) publicação nos últimos 10 anos.

Como critério de inclusão, utilizamos como parâmetro ter como variável a ser analisada a política pública desportiva local produzida pelo próprio município (descentralização bottomup). Para aquelas políticas advindas de níveis de governo superior (descentralização top-down), a política pública desportiva local poderia ser a variável dependente ou explicativa.

Descartamos os trabalhos (critério de exclusão) que: (a) retratassem a política desportiva educacional ${ }^{5}$; (b) se referissem a experiências de outros países que não Brasil e Portugal; (c) trabalhos que tratam do perfil do gestor público; e (d) de trabalhos que realizem diagnóstico de equipamentos desportivos fora da avaliação do papel da gestão na configuração dessa realidade.

A verificação da acurácia dos resultados exigiu ler o resumo dos trabalhos, a fim de poder identificar a ação do governo que foi analisada. Quando o resumo não permitia identificar os critérios de inclusão, estabelecemos o seguinte procedimento: (1) leitura da metodologia do trabalho; (2) leitura da íntegra do trabalho. Foram descartados 168 (70,59\%) artigos. A captura dos artigos foi encerrada em 30 em dezembro de 2019. Após a aferição da acurácia, restaram oito $(11,43 \%)$ trabalhos que retratam a realidade portuguesa e $62(88,57 \%)$, a realidade brasileira.

Após o levantamento bibliográfico que resultou na identificação de 70 trabalhos que contemplam todos os critérios aqui apresentados, elaboramos um banco de dados na planilha eletrônica Microsoft Excel (2010) contendo as seguintes informações: (a) a catalogação bibliográfica do artigo; (b) número de autores; (c) número de instituições envolvidas; (d) nome dos autores; (e) instituições envolvidas; (f) intercâmbio intrainstitucional, quando todos os autores eram de uma mesma instituição; (g) intercâmbio interinstitucional, quando

\footnotetext{
5 Tanto no Brasil como em Portugal as políticas de desporto educacional são fortemente impactadas pelas políticas educacionais, visto que o setor responsável pelas decisões políticas são os educacionais. No caso em pauta, nós estamos preocupados em conhecer aquelas produções associadas exclusivamente as políticas de esporte participação (para todos) e esporte de alto-rendimento (elite).
} 
apresentavam autores de mais de uma instituição dentro de um mesmo país; (h) intercâmbio internacional, quando apresentam parceria entre autores de mais de um país.

Há uma graduação entre as parcerias institucionais que respondem à seguinte fórmula: trabalho autoral < parceria intrainstitucional < parceria interinstitucional < parceria internacional. Sempre que houver mais de dois parceiros com níveis institucionais diferentes, será considerado aquele do maior nível. Para análise dos trabalhos, utilizou-se a estatística descritiva.

\section{Resultados e discussões}

Preliminarmente, buscamos identificar o tamanho das comunidades que ajudaram no desenvolvimento do campo de estudo com pelo menos um trabalho. No Brasil, essa comunidade é formada por 116 pesquisadores: 11 efetivos (9,48\%) e 105 eventuais (90,52\%). Em Portugal, encontramos 10 pesquisadores: três efetivos (30\%) e sete eventuais (70\%).

Essa primeira análise permite fazer algumas constatações importantes:

(a) mesmo diante da inexistência de parâmetros para avaliar a suficiência ou não do número de pesquisadores, os dois países juntos apresentam um estoque de 126 pesquisadores que possuem produção no campo da análise das políticas públicas desportivas municipais;

(b) a comunidade de pesquisadores brasileiros apresenta elevado número de pesquisadores eventuais, que é um indicativo de que o campo de investigação ainda não está consolidado;

(c) a comunidade de pesquisadores portugueses, apesar de menor, demonstra que há maior envolvimento em torno da agenda, pois $30 \%$ são de pesquisadores efetivos;

(d) como pode ser observado na tabela 1, o Brasil tem grau de diversificação institucional de $100 \%$ entre seus pesquisadores efetivos, pois cada um é oriundo de uma instituição. Em Portugal, dois são da Universidade do Porto e o outro não desenvolve atividade acadêmica;

(e) enquanto os pesquisadores efetivos do Brasil estão representados em 67,74\% dos trabalhos, os pesquisadores efetivos portugueses representam $87,5 \%$ das autorias;

(f) a segunda metade do período é responsável por 67,14\% dos trabalhos e 2019 responde por $28,57 \%$; 
Como pode ser observado na tabela 1 , dentre as duas comunidades se destaca a participação do Pesquisador 09 (P09) ${ }^{6}$ como aquele de maior contribuição no desenvolvimento do campo. O P13 é o segundo maior colaborador geral. Na terceira posição geral está a P04, que é a mais tem contribuído para o desenvolvimento do tema em Portugal. É perceptível a concentração na participação na produção dos trabalhos em apenas três pesquisadores, o que é um indicativo da fragilidade (ou imaturidade) do subcampo de investigação.

Uma comunidade de especialistas pressupõe a existência de múltiplos centros de produção. No caso do Brasil, são 10 instituições e pesquisadores envolvidos. Em Portugal o subcampo é menos diverso, são dois pesquisadores da mesma instituição e um sem vinculação com alguma instituição de nível superior.

Tabela 1. Ranking de autoria dos pesquisadores efetivos e instituições

\begin{tabular}{|c|c|c|}
\hline Brasil/Portugal & n & $\%$ \\
\hline P09 & 21 & 10,55 \\
\hline P13 & 12 & 6,03 \\
\hline P04 & 9 & 4,52 \\
\hline P06, P07, P10, P11 & 4 & 8,04 \\
\hline $\mathrm{P} 01, \mathrm{P} 02, \mathrm{P} 05, \mathrm{P} 08, \mathrm{P} 12, \mathrm{P} 14$ & 3 & 10,55 \\
\hline \multicolumn{3}{|l|}{ Brasil } \\
\hline P09 (UNIVASF) & 21 & 12,07 \\
\hline P13 (UEM) & 12 & 6,9 \\
\hline P07 (UFPE), P11(UFES) & 4 & 4,6 \\
\hline $\begin{array}{l}\text { P01 (UESC), P02 (USP), P05 (UTFPR), P08 (UEMT), } \\
\text { P12 (UESB), P14 (UFPR) }\end{array}$ & 3 & 12,07 \\
\hline \multicolumn{3}{|l|}{ Portugal } \\
\hline P04 (UP) & 9 & 36 \\
\hline P06 (s/I*), P10 (UP) & 4 & 32 \\
\hline
\end{tabular}

O primeiro autor representa o protagonismo da agenda e é capaz de retratar com maior precisão as virtudes e as limitações do campo. Os pesquisadores efetivos como primeiro autor são responsáveis por 45,71\% das produções (tabela 2). Apesar do volume de trabalhos para poucos pesquisadores, o subcampo tem se desenvolvido com contribuições esporádicas $(54,29 \%)$ que são insuficientes para constituir uma comunidade de especialistas.

Como pode ser observado na tabela 2, apenas cinco pesquisadores, dentre os pesquisadores efetivos, aparecem como primeiro autor com mais de duas publicações. E um

\footnotetext{
${ }^{6}$ Os pesquisadores efetivos foram agrupados, classificados por ordem alfabética do último nome: Ávila, M.A. (P 01); Bastos, F. D. C. (P 02); Borges, C. N. F (P 03); Carvalho, M. J. (P 04); Hirata, E. (P 05); Januário, C. (P 06); Menezes, V. G. (P 07); Reverdito, R. S. (P 08); Santos, E. S. (P 09); Sarmento, P. (P 10); Silva, D. S. (P 11); Silva, T. D. (P 12); Starepravo, F. A (P 13); Werle, V. (P 14).
} 
único autor (P09) é responsável por 65,63\% da produção como primeiro autor. No Brasil, ele responde por $77,78 \%$ da produção como primeiro autor. Em Portugal, P06 responde por $60 \%$ da produção dos efetivos como primeiro autor e P04, por 40\%. As evidências indicam que nos dois países a produção como primeiro autor nesta área está restrita a poucos pesquisadores. Outra evidência que aponta a fragilidade do subcampo.

Tabela 2. Ranking primeiro autor com mais de dois trabalhos publicados

\begin{tabular}{|c|c|c|}
\hline Brasil/Portugal & $\mathrm{n}$ & $\%$ \\
\hline P09 & 21 & 65,63 \\
\hline P06, P11, P14 & 3 & 28,13 \\
\hline P04 & 2 & 6,65 \\
\hline \multicolumn{3}{|c|}{ Brasil } \\
\hline P09 & 21 & 77,78 \\
\hline $\mathrm{P} 11, \mathrm{P} 14$ & 3 & 22,22 \\
\hline \multicolumn{3}{|c|}{ Portugal } \\
\hline P06 & 3 & 60 \\
\hline P04 & 2 & 40 \\
\hline
\end{tabular}

Fonte: Dados da pesquisa.

Como destaca Silva (2002), as redes científicas podem ser abertas e ágeis, permitindo maior intercâmbio e desenvolvimento do campo. Na mesma direção, Bulgacov \& Verdu (2001) compreendem que esses intercâmbios oferecem maiores oportunidades para o aprimoramento científico e tecnológico. Desta forma, das produções autorais até as parcerias internacionais, há uma graduação que permite compreender melhor o desenvolvimento de um determinado campo de investigação. Para tanto, temos que analisar com maior precisão aqueles que têm a agenda da investigação, comumente o primeiro autor.

A montagem da sequência de autoria de forma alfabética poderia não permitir avaliar a questão do primeiro autor. No entanto, essa composição pressupõe que os outros autores também tenham essa agenda como algo prioritário em sua produção. Considerando esses dois aspectos, não foi possível encontrar essa realidade entre os pesquisadores efetivos. Nesse caso, o primeiro autor representa o detentor da agenda de pesquisa.

O pesquisador que aparece com primeiro autor e que apresenta o maior nível de intercâmbio é P09. Sua produção é 100\% como primeiro autor, 76,19\% (16) das produções envolve algum tipo de intercâmbio interinstitucional e 12,28\% (2) são colaborações internacionais. Em suas relações interinstitucionais, seu maior parceiro é o segundo autor com maior número de autoria (tabela 1), P13, que responde por $50 \%$ de parceria da produção interinstitucional do pesquisador. 
Essa parceria tem permitido ampliar o leque de parceiros, com destaque para aqueles que aparecem como efetivos: P04, P05, P07, P08 e P13. Três dos parceiros (entre efetivos e eventuais) pertencem ao grupo de pesquisa coordenado pelo P13. Considerando que esses coautores estabeleceram relações de orientação com P13, essa aproximação tem permitido compartilhar a avaliação da agenda de pesquisa junto a um grupo de pesquisa. Como nenhum desses coautores se constituiu como primeiro autor de trabalhos do subcampo, há limitações importantes para o compartilhamento da agenda, fundamental para o desenvolvimento de qualquer campo de investigação. São parcerias que estão circunscritas ao aprimoramento crítico os trabalhos, tarefa não menos importante ao desenvolvimento de qualquer campo de investigação, mas insuficientes para os desafios do subcampo.

As parcerias internacionais do P09 são com a pesquisadora com maior participação na produção sobre o tema em Portugal (tabela 1), P04. Essa aproximação, da mesma forma que o grupo do P13, tem permitido incorporar outros pesquisadores de seu grupo, como $\mathrm{GF}^{7}$.

Considerando o leque de parcerias com diferentes autores e sua posição na articulação desse subcampo, o pesquisador se encaixa no conceito de betweenness centrality de Soares, Souza \& Moura (2010). A retirada de sua produção desse quadro, além de desidratar o subcampo, tornaria ainda mais frágil os intercâmbios. Essa é uma evidência importante que dificulta a constituição de um subcampo em torno da análise das políticas públicas de desporto municipal no atual contexto.

A mesma realidade é experimentada em Portugal. A P04 está em 75\% (6) dos textos que retratam a realidade portuguesa, é parceira de todos os trabalhos do P06 e desenvolve parcerias internacionais com o P09 e com o pesquisador moçambicano GP ${ }^{8}$. Apesar de não figurar como primeira autora na maior parte dos trabalhos, parece evidente também sua participação como betweenness centrality, com consequência similar ao P09.

Na tabela 3, é possível observar que os cinco pesquisadores efetivos com mais de dois trabalhos têm diferentes modos de produção do conhecimento. A pesquisadora P04 distribui sua produção em 50\% em parcerias intrainstitucionais e 50\% em parcerias internacionais. As parcerias intrainstitucionais estão associados ao trabalho de seu grupo de pesquisa que evolve produção com orientandos e parceiros da temática. Na parceria internacional se destaca aquela com o P09. Essa realidade desnuda uma das fragilidades da constituição de um subcampo em

\footnotetext{
${ }^{7}$ George Froes.

${ }^{8}$ Gustavo Paipe.
} 
Portugal, a inexistência do compartilhamento desta agenda de pesquisa com outras instituições. Outro pesquisador português, P06, também desnuda essa dificuldade, pois $100 \%$ de sua produção se dá no nível interinstitucional, muitas delas em parceria com P04.

A produção de P09 é a mais diversificada do grupo, ela está distribuída em todos os níveis. Responde por 83,3\% (5) das produções autorais. A baixa produção interinstitucional se deve pelo fato de o autor não participar de programa de pós-graduação, fato que, talvez, tenha o tornado dependente das parcerias interinstitucional e internacional. As parcerias interinstitucionais se dão (38\%) com P13 que, além de ser uma das referências em análise de políticas públicas no Brasil, tem um grupo forte dentro do programa de pós-graduação. Percebese claramente a estratégia de construir parcerias com outros pesquisadores de outras instituições. A parceria internacional se dá exclusivamente com o centro de maior produção sobre a temática em Portugal.

Quando se observa a trajetória de P11, é possível perceber que sua produção interinstitucional acompanha seu processo de formação aliada a parceiros que manteve durante esse processo. Da mesma forma se dá a produção de P14, vinculada aos parceiros construídos durante o processo de formação.

Tabela 3. Nível de intercâmbio institucional dos pesquisadores efetivos com mais de dois

\begin{tabular}{|c|c|c|c|c|c|c|c|c|c|c|}
\hline \multirow{2}{*}{$\begin{array}{c}\text { Nível } \\
\text { Intercâmbio }\end{array}$} & \multicolumn{2}{|c|}{ P04 } & \multicolumn{2}{|c|}{ P06 } & \multicolumn{2}{|c|}{ P09 } & \multicolumn{2}{|c|}{ P11 } & \multicolumn{2}{|c|}{ P14 } \\
\hline & $\mathbf{n}$ & $\%$ & $\mathbf{n}$ & $\%$ & $\mathbf{n}$ & $\%$ & $\mathbf{n}$ & $\%$ & $\mathbf{n}$ & $\%$ \\
\hline Autoral & 0 & 0 & 0 & 0 & 5 & 23,8 & 0 & 0 & 1 & 33,3 \\
\hline Intrainstitucional & 5 & 50 & 4 & 100 & 1 & 4,8 & 0 & 0 & 1 & 33,3 \\
\hline Interinstitucional & 0 & 0 & 0 & 0 & 12 & 57,1 & 4 & 100 & 1 & 33,3 \\
\hline Internacional & 5 & 50 & 0 & 0 & 3 & 14,3 & 0 & 0 & 0 & 0 \\
\hline
\end{tabular}

Fonte: Dados da pesquisa.

Prevalece empreendimento coletivo neste subcampo de investigação, que é fundamental para o desenvolvimento científico (Watanabe, Gomes \& Hoffmann, 2013; Gatti, 2005; Bulgacov \& Verdu, 2001; Balancieri et al, 2005). Para 85,7\% das produções ocorrem parcerias intrainstitucional, interinstitucional e internacional. Diante da fragilidade do subcampo, principalmente pela existência dos betweenness centrality, essa estratégia aponta para uma possível consolidação futura da agenda de pesquisa. 
Nos dois países, Brasil e Portugal, a maior parte da produção de políticas desportivas no nível do poder local está concentrada num nível muito restrito de pesquisadores efetivos. Portanto, há uma agenda de pesquisa ainda por ser desenvolvida e baixo número de pesquisadores efetivos. Essa realidade não é suficiente para constituição do subcampo de investigação específico, apesar da especificidade da agenda e da lacuna informacional.

Passados mais de 30 anos da promulgação das duas constituições e da participação dos municípios na formulação e implementação de políticas públicas desportivas, um longo caminho precisa ser percorrido para aprimorar nossa compreensão do papel do poder local na oferta de políticas públicas desportivas. O contexto aqui descrito dificulta a produção de uma agenda de pesquisa sólida, pois ela só pode se efetivar em rede e com maior densidade de análises (Arretche, 2003; Reis, 2003).

\section{Conclusão}

No Brasil e em Portugal, há grande oferta de políticas desportivas pelo poder público no âmbito local. Os municípios, no caso do Brasil, e as câmaras municipais, no caso de Portugal, têm realizados gastos importantes em políticas públicas desportivas. Portanto, há uma agenda a ser explorada que contrasta com o pequeno número de pesquisadores efetivos nos dois países.

Dentre os pesquisadores efetivos, há diferentes comportamentos em relação à agenda $\mathrm{e}$ às parcerias institucionais. A detenção da agenda, que é um aspecto importante, acabou desnudando uma das fragilidades do subcampo. Não há uma rede de pesquisadores em torno da agenda, mas uma rede de colaboradores que têm cumprido papel importante no seu aprimoramento.

As redes de colaboração científica criadas são tênues e envolvendo baixo número de pesquisadores. Não há o compartilhamento de agenda de investigação. As colaborações se dão no nível do aprimoramento acadêmico científico das produções. Apesar de relevante e necessário ao desenvolvimento do campo, esse comportamento não é suficiente para sua constituição como uma agenda específica de investigação.

Os principais personagens são betweenness centrality, o que impõe certas limitações ao subcampo. No entanto, é perceptível o esforço na consolidação da agenda: o aumento do trabalho ao longo do tempo, os grupos de pesquisadas envolvidos e o número de parcerias interinstitucionais e internacionais. Portanto, há uma base estruturada que será fundamental para o maior envolvimento e aprimoramento do subcampo. 


\section{Referências}

Amaral, S. C. F., \& Pereira, A. P. C. (2009). Reflexões sobre a produção em políticas públicas de educação física, esporte e lazer. Revista Brasileira de Ciências do Esporte, 31(1), 41-56.

Amaral, S. C. F., Ribeiro, O. C. F., \& Silva, D. S. (2014). Produção científico-acadêmica em Políticas Públicas de Esporte e Lazer no Brasil. Motrivivência, 26(42), 27-40.

Araújo, C. A. (2006). Bibliometria: evolução histórica e questões atuais. Em questão, 12(1), 11-32.

Arretche, M. (2003). Dossiê agenda de pesquisa em políticas públicas. Revista Brasileira de Ciências Sociais, 18(51), 7-9.

Balancieri, R., Bovo, A., Kern, V., Pacheco, R. \& Barcia, R. (2005). A análise de redes de colaboração científica sob as novas tecnologias de informação e comunicação: um estudo na Plataforma Lattes. Ciência da Informação, 31(1), 64-77.

Barra, A. D. O., Silva, S. A. P. D. S., \& Vitorino, M. R. (2017). Panorama dos grupos de pesquisa em políticas públicas de esporte e lazer no Brasil presentes no diretório de grupos de pesquisa do CNPQ. Licere, 20(1), 38-59.

Bastos, F.C. (2003). Administração Esportiva: área de estudo, pesquisa e perspectivas no Brasil. Motrivivência, (20-21), 295-306.

Bulgacov, S., \& Verdu, F. C. (2001). Redes de pesquisadores da área de administração: um estudo exploratório. Revista de Administração Contemporânea, 5(SPE), 163-182.

CAPES. Portal.periódicos.capes. Disponível em: 〈http://www.periodicos. capes.gov.br> Acesso em: 18 nov. 2019.

Caregnato, S. E. (2011). Google Acadêmico como ferramenta para os estudos de citações: Avaliação da Precisão das Buscas por Autor. Ponto de Acesso, 5(3), 72-86.

Castro, S. B. E., de Lima Moro, N. R. N., da Silveira, L. R. T., \& Mezzadri, F. M. (2012). O estado da arte em políticas sociais de esporte e lazer no Brasil (2000-2009). Pensar a Prática, 15(2), 531-545.

Gatti, B. A. (2005). Formação de grupos e redes de intercâmbio em pesquisa educacional: dialogia e qualidade. Revista Brasileira de Educação, 30, 124-132.

Gazda, E., \& Quandt, C. O. (2010). Colaboração interinstitucional em pesquisa no Brasil: tendências em artigos na área de gestão da inovação. RAE-eletrônica, 9(2).

Giustini D. \& Barsky E. (2005). A look at Google Scholar, PubMed, and Scirus: comparisons and recommendations. Journal of the Canadian Health Libraries Association, 26(3), 85-89. 
Gregori Júnior, F. G., de Godoy, M. F., \& Gregori, F. F. (2012). Proposta de um índice cientométrico individual, com ênfase na ponderação positiva da participação do primeiro autor: índice H-FAC. Revista Brasileira de Cirurgia Cardiovascular/Brazilian Journal of Cardiovascular Surgery, 27(3), 370-376.

Hoekman, R, Jan-Willem van der Roest \& Hugo van der Poel (2018) From welfare state to participation society? Austerity measures and local sport policy in the Netherlands, International Journal of Sport Policy and Politics, 10(1), 131-146.

Januário, C. (2010) Políticas públicas desportivas: estudo centrado nos municípios da área metropolitana do Porto. Universidade do Porto, Porto, Portugal.

Kocian, R. C., \& da Silva, C. L. (2016). Políticas públicas e os equipamentos esportivos de lazer: produção acadêmica de 2008 a 2014. Licere, 19(3), 185-206.

Kuhn, T. (1976), A estrutura das revoluções científicas. São Paulo, Perspectiva.

Maia, M. D. F. S., \& Caregnato, S. E. (2008). Co-autoria como indicador de redes de colaboração científica. Perspectivas em Ciência da Informação, 13(2), 18-31.

Onofre, T., Colângelo, J. V. M., \& Lino, W. (2019). Balanço bibliométrico da produção científica em políticas públicas de lazer-Brasil 2012/2017. Revista Brasileira de Ciência e Movimento, 27(1), 164-176.

Paipe, G. P. (2017). Políticas públicas desportivas. Estudo centrado em municípios de Moçambique. (Tese Doutorado). Universidade do Porto, Porto, Portugal.

Petroianu, A. (2003). Critérios quantitativos para analisar o valor da publicação de artigos científicos. Rev Assoc Med Bras, 49(2), 173-6.

Ramchandani, G., Shibli, S., \& Kung, S. P. (2018). The performance of local authority sports facilities in England during a period of recession and austerity. International Journal of Sport Policy and Politics, 10(1), 95-111.

Reid, G. (2018). Managing budget cuts in Edinburgh's sport and recreation services: progressive localism in a resilient local authority? International Journal of Sport Policy and Politics, 10(1), 113-129.

Reis, E. (2000). Reflexões leigas para a formulação de uma agenda de pesquisa em políticas públicas. Red Revista Brasileira de Ciências Sociais, 18 (51): 21-30.

Sampaio, R. F. \& Mancini, M. C. (2007). Estudos de revisão sistemática: um guia para síntese criteriosa da evidência científica. Revista Brasileira de Fisioterapia, 11(1), 83-89.

Santos, A. L. F., Batista, M. C. A. \& Araújo, A. V. (2007). A produção do conhecimento em política pública para educação física, esporte e lazer: o GTT 10 em Pernambuco. In Anais do XV Congresso Brasileiro de Ciências do Esporte (pp 1-10). Recife. 
Santos, E. S. \& Freitas, A. L. C. (2015). O direito ao desporto: justiça social e políticas públicas. Direitos Fundamentais \& Justiça, 9, 139-157.

Santos, E. S., Milani, F. G., Reverdito, R. S. \& Starepravo, F. A. (2019). O financiamento do esporte e do lazer pelos municípios do estado do Mato Grosso de 2005 A 2008. Licere, 22(1), 49-69.

Silva, E. L. (2002). Rede científica e a construção do conhecimento. Informação \& Sociedade, 12(1).

Soares, G. A. D., Souza, C. P. R. D., \& Moura, T. W. D. (2010). Colaboração na produção científica na Ciência Política e na Sociologia brasileiras. Sociedade e Estado, 25(3), 525538.

Sousa, D. P., Gabriel, B. J., Antunes, A. C., Pedroso, B., de Oliveira Junior, C. R., \& de Freitas Jr, M. A. (2016). As produções do periódico "Pensar a Prática" que relacionaram os seus objetos às políticas públicas de esporte e lazer (1998-2015). Pensar a Prática, 19(3), 612-626.

Starepravo, F. A., Afonso, G., \& Ferreira, A. L. P. Produção do GTT de políticas públicas no XV Congresso Brasileiro de Ciências do Esporte: possibilidades teóricas de análise das políticas públicas de esporte e lazer. In Anais $1^{\circ}$ Encontro da ALESDE (pp 1-8). Curitiba.

Shultz, M. (2007). Comparing test searches in PubMed and Google Scholar. Journal of the Medical Library Association, 95(4), 442.

Watanabe, E. A., Gomes, A. O., \& Hoffmann, V. E. (2013). Cooperação entre grupos de pesquisa em estratégia no Brasil. Revista Ibero Americana de Estratégia, 12(1), 84-106. 